\title{
High-temperature quantum Hall effect in finite gapped HgTe quantum wells
}

\author{
T. Khouri, ${ }^{1, *}$ M. Bendias, ${ }^{2}$ P. Leubner, ${ }^{2}$ C. Brüne, ${ }^{2}$ H. Buhmann, ${ }^{2}$ L. W. Molenkamp, ${ }^{2}$ \\ U. Zeitler, ${ }^{1}$ N. E. Hussey, ${ }^{1}$ and S. Wiedmann ${ }^{1, \dagger}$ \\ ${ }^{1}$ High Field Magnet Laboratory (HFML-EMFL), Radboud University, Nijmegen, The Netherlands \\ ${ }^{2}$ Physikalisches Institut (EP3), Universität Würzburg, Am Hubland, 97074 Würzburg, Germany \\ (Received 10 December 2015; revised manuscript received 2 March 2016; published 18 March 2016)
}

\begin{abstract}
We report on the observation of the quantum Hall effect at high temperatures in HgTe quantum wells with a finite band gap and a thickness below and above the critical thickness $d_{\mathrm{c}}$ that separates a conventional semiconductor from a two-dimensional topological insulator. At high carrier concentrations, we observe a quantized Hall conductivity up to $60 \mathrm{~K}$ with energy gaps between Landau levels of the order of $25 \mathrm{meV}$, in good agreement with the Landau level spectrum obtained from $\mathbf{k} \cdot \mathbf{p}$ calculations. Using the scaling approach for the plateau-plateau transition at $v=2 \rightarrow 1$, we find the scaling coefficient $\kappa=0.45 \pm 0.04$ to be consistent with the universality of scaling theory, and we do not find signs of increased electron-phonon interaction to alter the scaling even at these elevated temperatures. Comparing the high-temperature limit of the quantized Hall resistance in $\mathrm{HgTe}$ quantum wells with a finite band gap with room-temperature experiment in graphene, we find that the energy gaps at the breakdown of the quantization exceed the thermal energy by the same order of magnitude.
\end{abstract}

DOI: 10.1103/PhysRevB.93.125308

\section{INTRODUCTION}

The quantum Hall effect (QHE) [1] is a universal phenomenon that occurs when two-dimensional (2D) metallic systems are subjected to a perpendicular magnetic field. The magnetic field splits the constant density of states into discrete Landau levels (LLs), which are separated by energy gaps $\Delta E$. When the Fermi energy $E_{\mathrm{F}}$ is in the gapped regions, the Hall conductance is quantized to integer multiples of $e^{2} / h$, where $h$ is Planck's constant and $e$ is the electron charge. This quantization has been observed in a wide range of semiconductor heterostructures, such as GaAs/AlGaAs, Si-MOSFETs, and SiGe [2-4]. The origin of the QHE can be explained on the basis of localized and extended states that occur in the spectrum of impurity-broadened LLs [5,6]. In the center of the LLs, extended states exist that lead to a metallic behavior, while in the vicinity of localized states the bulk behavior is insulating. Although bulk states in between LLs are localized, dissipationless 1D edge channels are formed that dominate the transport properties in this regime. The consequence is a quantized Hall resistance accompanied by a vanishing longitudinal resistance. To observe this quantization, low temperatures $(T \lesssim 10 \mathrm{~K})$ are, in general, necessary to prevent thermal occupation of extended states in higher LLs. This limit has recently been overcome by a new class of systems that exhibit a linear dispersion. One of the most famous systems with a linear dispersion is the zero-gap semiconductor graphene, where the QHE has been observed at room temperature using a magnetic field of $29 \mathrm{~T}$ [7]. The observation of a quantized resistance at these high temperatures is based on the peculiar nature of charge-carrier quantization in a magnetic field, which is given by $E_{N}= \pm v_{\mathrm{F}} \sqrt{2 e \hbar B N}$, where $v_{\mathrm{F}}$ is the Fermi velocity, $B$ is the magnetic field, $\hbar$ is the reduced Planck constant, and $N$ is the Landau level index. For a typical Fermi velocity in graphene of $v_{\mathrm{F}} \approx 10^{6} \mathrm{~m} / \mathrm{s}$,

\footnotetext{
*T.Khouri@science.ru.nl

${ }^{\dagger}$ S.Wiedmann@science.ru.nl
}

the energy gap between the lowest LL $(N=0)$ and the first excited one $(N=1)$ is $\Delta E \approx 195 \mathrm{meV}$ at $29 \mathrm{~T}$, exceeding the thermal energy at room temperature $\left(k_{\mathrm{B}} T \approx 25 \mathrm{meV}\right)$ by almost one order of magnitude. Unlike in conventional systems, where the second subband is relatively close to the first, the second subband in graphene is not occupied up to very high temperatures, thereby supporting the condition for the observation of the QHE.

Similar conditions are present in HgTe quantum wells (QWs). A remarkable property of these so-called type-III QWs is that by tuning the quantum-well thickness $d_{\mathrm{QW}}$ above a critical thickness $d_{\mathrm{c}}=6.3 \mathrm{~nm}$, a transition from a semiconductor to a topological insulator (TI) is achieved [8]. At $d_{\mathrm{QW}}=d_{\mathrm{c}}$, the conduction and valence bands touch each other, which leads to a single-valley gapless 2D Dirac-fermion system [9,10], and the QHE can be observed up to nitrogen temperatures [11]. In contrast to conventional semiconductors, $\mathrm{HgTe}$ QWs with a finite (bulk) band gap also have a highly nonparabolic dispersion, approaching a linear energy-momentum relation at finite $k$, and they are described by the Dirac Hamiltonian [12]. The presence of a small but finite bulk band gap affects the LL dispersion and consequently the energy gaps in the LL spectrum compared to zero-gap systems [11].

In this paper, we investigate the high-temperature $\mathrm{QH}$ regime of HgTe quantum wells with a finite band gap with $d_{\mathrm{OW}}$ below and above $d_{\mathrm{c}}$. The combination of the dispersion relation, leading to charge carriers that obey the Dirac equation, with a second subband that is more than $100 \mathrm{meV}$ above $E_{\mathrm{F}}$ makes our system ideal to study the QHE up to high temperatures with only one occupied subband.

\section{SAMPLE CHARACTERIZATION}

Our samples were grown by molecular beam epitaxy (MBE) in the [001] direction, and they were structured into Hall bars of dimensions $L \times W$ of $(600 \times 200)$ and $(30 \times 10) \mu \mathrm{m}^{2}$, respectively. The quantum-well thicknesses of the two samples are $d_{\mathrm{QW}}=5.9 \mathrm{~nm}$ (sample 1) and $d_{\mathrm{QW}}=11 \mathrm{~nm}$ (sample 2), respectively. The calculated energy dispersions $E(k)$ using a 
$\mathbf{k} \cdot \mathbf{p}$ model with an $8 \times 8$ Kane Hamiltonian [13] are shown in Figs. 1(a) and 1(b), where the first $\left(E_{1}\right)$ and second $\left(E_{2}\right)$ electron- and holelike $\left(H_{1}\right.$ and $\left.H_{2}\right)$ subbands are plotted. Both systems possess a finite bulk band gap, and while sample 1 is a trivial semiconductor with a direct band gap of $12 \mathrm{meV}$, sample 2 is a 2D TI with an indirect band gap of $3 \mathrm{meV}$ and an inverted band ordering giving rise to helical edge states at zero magnetic field $[8,14]$. This difference can be seen experimentally when tuning the Fermi energy $E_{\mathrm{F}}$ with the top gate through the band gap while measuring the low-temperature $(T=4.2 \mathrm{~K})$ longitudinal resistance $R_{x x}$ [see Figs. 1(c) and 1(d)]. Compared to the expected insulating behavior of sample $1\left(R_{x x} \geqslant 1 \mathrm{M} \Omega\right)$, sample 2 has a significantly reduced resistance $\left(R_{x x} \approx 27 \mathrm{k} \Omega\right)$ when $E_{\mathrm{F}}$ is in the bulk band gap. This value is above the expected quantization for a 2D TI due to the formation of charge puddles in larger samples ( $L \gtrsim 1 \mu \mathrm{m})$, which can lead to backscattering $[8,15,16]$ as well as the thermal activation of bulk carriers over the narrow band gap of $3 \mathrm{meV}$.

To study the QHE at high temperatures, we tune the Fermi energy $E_{\mathrm{F}}$ deep into the conduction band where the dispersion is nearly linear. We achieve this by applying gate voltages of $V_{g, 1}=1 \mathrm{~V}$ (sample 1) and $V_{g, 2}=1.4$ $\mathrm{V}$ (sample 2), which yields almost equal charge-carrier concentrations of $n_{\mathrm{s}, 1}=4.59 \times 10^{11} \mathrm{~cm}^{-2}$ and $n_{\mathrm{s}, 2}=4.66 \times$ $10^{11} \mathrm{~cm}^{-2}$, respectively. In this regime, our samples have mobilities of $\mu_{1}=67800 \mathrm{~cm}^{2} / \mathrm{V} \mathrm{s}$ and $\mu_{2}=82400 \mathrm{~cm}^{2} / \mathrm{V} \mathrm{s}$, as determined from the zero-field resistivity. For both carrier
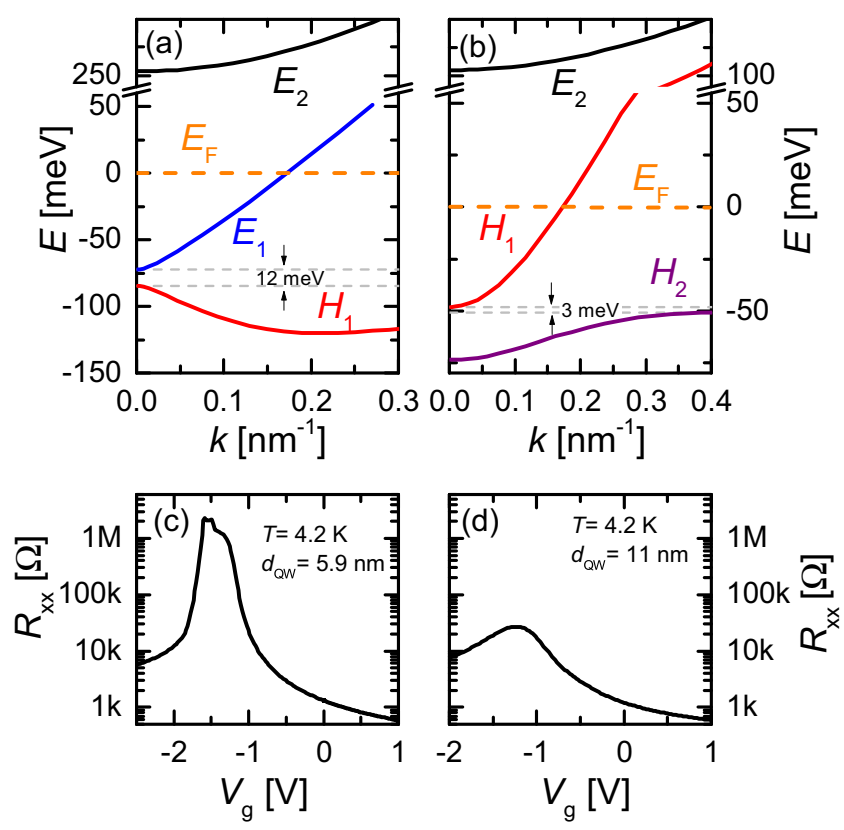

FIG. 1. Band-structure calculations performed with the $\mathbf{k} \cdot \mathbf{p}$ model using an $8 \times 8$ Kane-Hamiltonian for (a) a 5.9-nm-thick and (b) an 11-nm-thick HgTe QW. Here the lowest two electronlike ( $E_{1}$ and $\left.E_{2}\right)$ and holelike $\left(H_{1}\right.$ and $\left.H_{2}\right)$ subbands are shown. The Fermi energy at electron densities where $\mathrm{QH}$ experiments were performed (see Fig. 2) of (a) $n_{s 1}=4.59 \times 10^{11} \mathrm{~cm}^{-2}$ and (b) $n_{s 2}=4.66 \times$ $10^{11} \mathrm{~cm}^{-2}$ is marked by dashed lines. These densities correspond to constant gate voltages of $V_{g, 1}=1 \mathrm{~V}$ and $V_{g, 2}=1.4 \mathrm{~V}$, respectively. Gate-dependent measurements at $T=4.2 \mathrm{~K}$ of the longitudinal resistance $R_{x x}$ for the (c) 5.9-nm-thick and (d) 11-nm-thick samples. concentrations, $E_{\mathrm{F}}$ is more than $100 \mathrm{meV}$ below the second electronic subband $E_{2}$, as can be seen in Figs. 1(a) and 1(b), from which we exclude thermal excitation of higher subbands contributing to transport in our experiment. The nearly linear dispersion in combination with a single occupied subband up to high energies are perfect conditions for the observation of the QHE at high temperatures.

For the experiment, we used standard four-terminal lock-in techniques and carefully chose our excitation to prevent heating of the samples. We used a ${ }^{3} \mathrm{H}$-system to access a temperature range from 0.3 to $80 \mathrm{~K}$ in magnetic fields up to $30 \mathrm{~T}$.

\section{RESULTS AND DISCUSSION}

The main results of our measurements are summarized in Fig. 2, where we present data that represent the overall behavior of our samples. Figures 2(a) and 2(b) show measurements of $R_{x x}$ of the two samples at constant charge-carrier concentrations and different temperatures. From additional measurements of $R_{x y}$ and the known geometry of the Hall bars, we determine the corresponding resistivities $\rho_{x x}$ and $\rho_{x y}$ from which we calculate the Hall conductivities $\sigma_{x y}=$ $\rho_{x y} /\left(\rho_{x y}^{2}+\rho_{x x}^{2}\right)$ plotted in Figs. 2(c) and 2(d). The insets show a magnification of the region around filling factor $v=1$, where $v$ is defined as $v=n_{\mathrm{s}} / n_{\mathrm{L}}=n_{\mathrm{s}} h / e B$, where $n_{\mathrm{s}}$ is the charge-carrier concentration and $n_{\mathrm{L}}$ is the degeneracy of the Landau levels.

We observe pronounced Shubnikov-de Haas oscillations in the displayed temperature range accompanied by plateaus in $\sigma_{x y}$ at $v=1$ up to 60 and $46.5 \mathrm{~K}$ for samples 1 and 2, respectively. From the temperature dependence of the minima in $R_{x x}$, we extract the activation gaps $\Delta E$ between adjacent LLs with a Fermi-Dirac fit, and we compare the results with theoretical calculations of the Landau level dispersions shown in Figs. 3(a) and 3(b). In contrast to the LL fan chart of the 5.9 $\mathrm{nm}$ quantum well where all LLs show a positive dispersion, the inverted sample exhibits a LL crossing of one electronlike and one holelike level at around $8 \mathrm{~T}$, which is the hallmark of a 2D inverted system [9].

The experimentally and theoretically obtained energy gaps are in reasonably good agreement, as shown in Figs. 3(c) and 3(d), and the overall behavior of the sample is well described by our $\mathbf{k} \cdot \mathbf{p}$ model. While the calculations yield energy gaps of $\Delta E_{1, v=1} \simeq 46 \mathrm{meV}$ at $B \simeq 20 \mathrm{~T}$ and $\Delta E_{2, v=1} \simeq$ $39 \mathrm{meV}$ at $B \simeq 21 \mathrm{~T}$ for samples 1 and 2 , respectively, the extracted activation energies are slightly smaller and determined to be $\Delta E_{1, v=1} \simeq(42 \pm 1.5) \mathrm{meV}$ and $\Delta E_{2, v=1} \simeq$ (34 \pm 2.9$) \mathrm{meV}$. This small difference can mainly be attributed to the simplicity of our calculations, where we assume an infinitely small LL width. In reality, scattering from impurities or dopants leads to a broadening of the LLs, resulting in smaller energy gaps than our theoretical estimates, as observed. Despite the broadened LLs, the energy gap for the lowest filling factor still exceeds the thermal energy at room temperature of $k_{\mathrm{B}} T \approx 25 \mathrm{meV}$. We note furthermore that the energy gaps are larger than in conventional 2D systems, but they are still almost an order of magnitude smaller than in graphene due to a smaller Fermi velocity $\left(\simeq 5 \times 10^{5} \mathrm{~m} / \mathrm{s}\right)$ [17-19] compared to graphene but with a large Zeeman splitting $\Delta E_{\mathrm{Z}}=g^{*} \mu_{\mathrm{B}} B$ of 

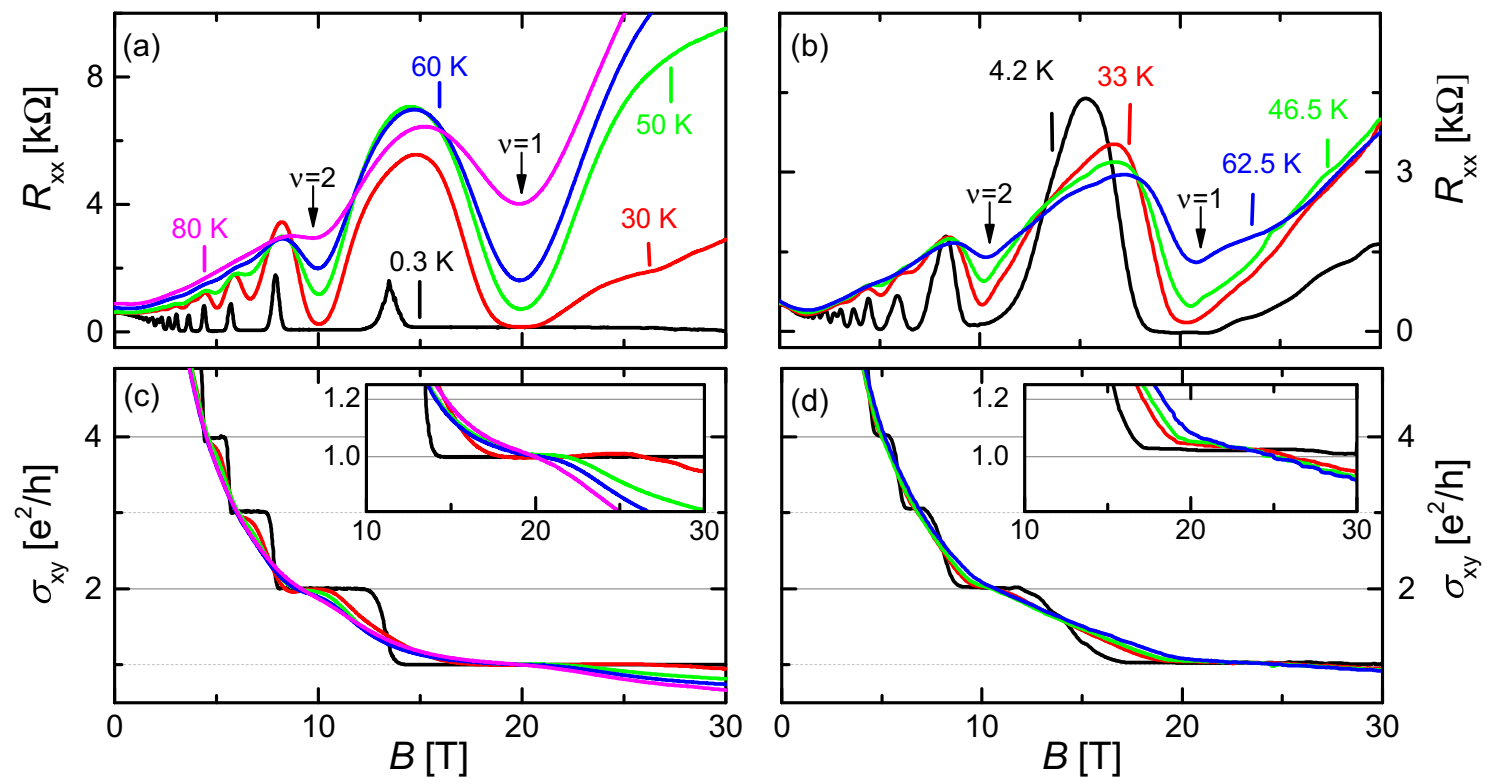

FIG. 2. Measurements of the longitudinal resistances $R_{x x}$ of the (a) 5.9-nm-thick and (b) 11-nm-thick QWs at constant electron densities $n_{\mathrm{s}, 1}=4.59 \times 10^{11} \mathrm{~cm}^{-2}$ and $n_{\mathrm{s}, 2}=4.66 \times 10^{11} \mathrm{~cm}^{-2}$ at different temperatures. The black arrows mark the position in magnetic field of filling factors $v=1$ and 2. In (c) and (d), the corresponding Hall conductivities $\sigma_{x y}$ are shown. The insets show a magnification of $\sigma_{x y}$ at filling factor 1 .
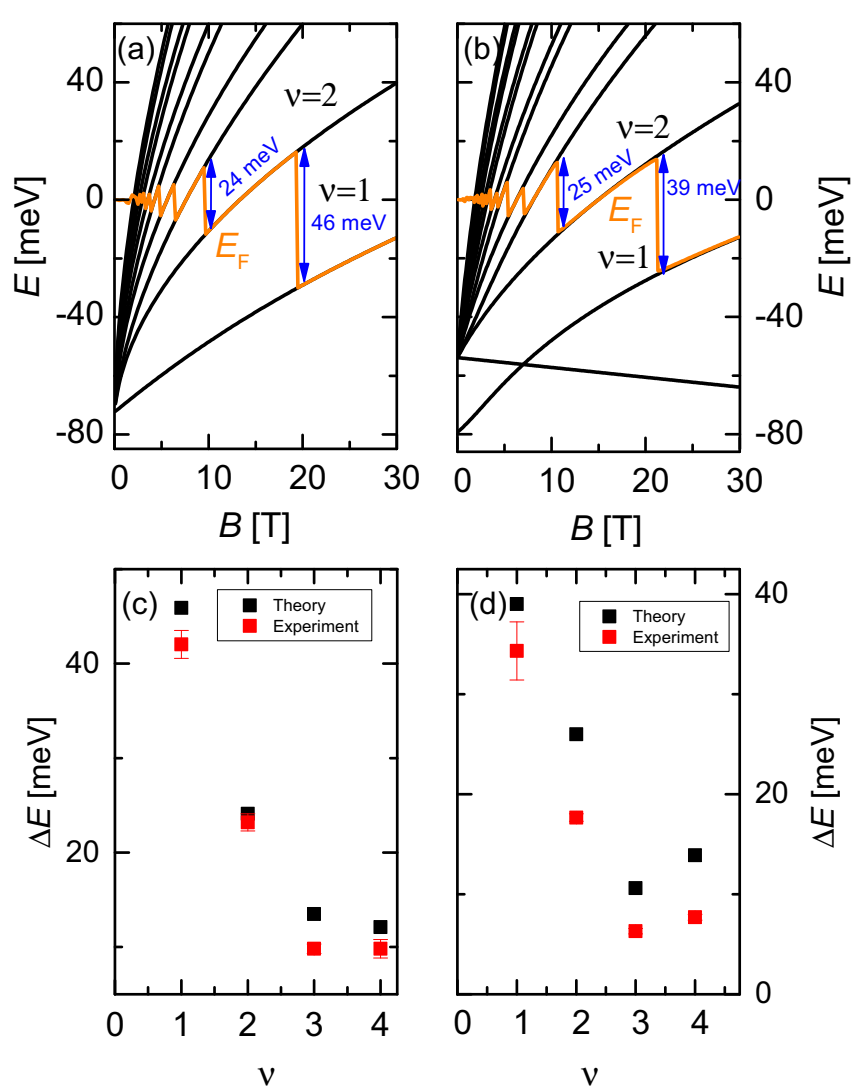

FIG. 3. Calculated LL dispersion for (a) a 5.9-nm-thick and (b) an 11-nm-thick HgTe quantum well. The position of the Fermi energy $E_{\mathrm{F}}$ is marked by the orange line. In (c) and (d), the corresponding experimentally extracted and theoretical calculated energy gaps are plotted for the lowest four filling factors. the LLs in HgTe, where $g^{*}$ is the effective Landé factor and $\mu_{\mathrm{B}}$ is the Bohr magneton. Therefore, the energy gap for the filling factor $v=1$ is $\Delta E_{v=1}=v_{\mathrm{F}} \sqrt{2 \hbar e B}-\Delta E_{\mathrm{Z}}$, and for $v=2$ it is $\Delta E_{v=2}=\Delta E_{\mathrm{Z}}$. At the Fermi energy, the Landau-level dispersions are very similar, and $g^{*} \simeq 20$ for both samples. A further increase in charge-carrier densities and magnetic-field range would, due to the nature of the LL dispersion, only slightly increase the energy gaps, and the maximum $\Delta E$ remains in the order of $50 \mathrm{meV}$. Thus, the temperature range where we still observe the QHE is largely reduced compared to graphene. Interestingly, the ratio of the energy gap $\Delta E$ to the thermal energy where $\sigma_{x y}$ is still quantized is comparable to the ratio of $\Delta E / k_{\mathrm{B}} T \approx 8$ measured in graphene.

Although our system has large energy gaps between adjacent LLs, a necessary but not sufficient condition for the observation of QHE at high temperatures, we need to consider the localization effects of charge carriers. In disordered systems, charge carriers in the tails of the LLs are localized, while extended states exist only at the center of each LL. Because of these localized states, the Fermi energy moves smoothly through the energy gap, and a plateaulike Hall resistance is observed in the measurements. A widely used approach to study localization is to investigate the scaling behavior of the unique insulator-metal transition, which occurs when the energy crosses from localized to delocalized states [20,21]. Within the finite-size scaling theory [20], it is possible to observe scaling behavior in the temperature dependence of the slope of the plateau transition; specifically, the maximum of the derivative of the Hall resistance scales with the temperature as

$$
\left(d R_{x y} / d B\right)^{\max } \propto T^{-\kappa},
$$

with $\kappa=p / 2 \gamma$, with $\gamma$ the critical localization length exponent and $p$ the scattering exponent. The same power-law 


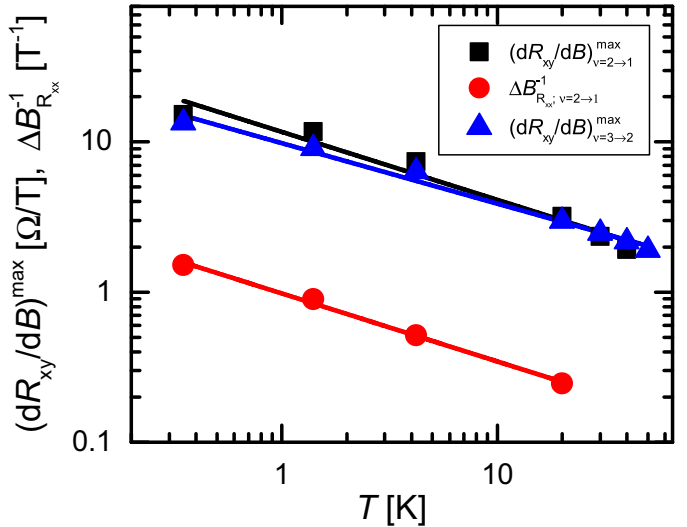

FIG. 4. Temperature dependence of the maxima of $\left(d R_{x y} / d B\right)$ and the FWHM of the $R_{x x}$ peaks $\Delta B_{R_{x x}}$ for the 5.9-nm-thick sample. From the slope of the linear fits, $\kappa=p / 2 \gamma$ can be determined with $\left(d R_{x y} / d B\right)^{\max } \propto \kappa$ and $\Delta B_{R_{x x}} \propto-\kappa$.

dependence holds for the temperature dependence of the full width at half-maximum (FWHM) of the $R_{x x}$ peaks, which we denote as $\Delta B_{R_{x x}}$. While the universal scaling theory predicts $\kappa$ and $p$ to be universal, it is still a controversial topic within the literature [22-25]. Since we are able to access a wide temperature range in which we still observe a quantized Hall conductivity, it is interesting to compare this scaling behavior with previous studies.

As shown in Fig. 4, the analysis of sample 1 of $\left(d R_{x y} / d B\right)^{\max }$ yields $\kappa=0.45 \pm 0.04$ for the transition from $v=2 \rightarrow 1$, in good agreement with the value $\kappa=0.45 \pm$ 0.02 extracted from $\Delta B_{R_{x x}}$. For the $v=3 \rightarrow 2$ transition, $\left(d R_{x y} / d B\right)^{\max }$ yields $\kappa=0.40 \pm 0.02$ (unfortunately there are not enough data points available for the analysis of $\Delta B_{R_{x x}}$ for this transition as the minima quickly rise above zero). Assuming $\gamma$ to be universal, we obtain $p=2.1 \pm 0.2$. All our values fit within the theory of universal scaling, suggesting that our system is described by short-range scattering [26]. Furthermore, there is no sign of increased electron-phonon interaction, which we expect to be present above $10 \mathrm{~K}$ and which would lead to a different scaling behavior [27,28]. Our scaling analysis at elevated temperatures is consistent with measurements on graphene [29] and shows no difference from that obtained on conventional 2D systems. Similar scaling analysis for sample 2 was not conclusive due to the large error bars in the obtained values of $\kappa$ and $p$. The scaling for a 20.3-nm-wide quantum well has recently been published, and the principal feasibility of scaling analysis in $\mathrm{HgTe}$ was addressed [30].

\section{SUMMARY}

In summary, we have studied the QHE in HgTe QWs with a finite band gap above and below the critical thickness $d_{\mathrm{c}}$ up to temperatures of the order of $50 \mathrm{~K}$. From temperaturedependent magnetotransport measurements, we extract energy gaps between LLs of the order of $25 \mathrm{meV}$. The thermal energy at which the Hall conductance is still quantized is almost a factor of 8 higher than the energy gap itself, showing striking similarities to graphene. We did not find any evidence of increased electron-phonon interaction that would alter the scaling behavior of the QHE between $v=1$ and 2. From the observed scaling, we determined $\kappa=0.45 \pm 0.04$ for the noninverted sample with $d_{\mathrm{QW}}=5.9 \mathrm{~nm}$, in excellent agreement with the universal scaling theory. An interesting subject for further theoretical and experimental studies is whether the high-temperature limit of the QHE is influenced by a difference in localization strength and can be related to sample disorder or mobility $\mu$.

\section{ACKNOWLEDGMENTS}

This work was performed at the HFML-RU/FOM member of the European Magnetic Field Laboratory (EMFL), and it was supported by EuroMagNET II and is part of the research programme of the Foundation for Fundamental Research on Matter (FOM), which is part of the Netherlands Organisation for Scientific Research (NWO). The Würzburg group would like to acknowledge funding from the German Research Foundation (The Leibniz Program, Sonderforschungsbereich 1170 Tocotronics and Schwerpunktprogramm 1666), the EU ERC-AG program (Project 3-TOP), and the Elitenetzwerk Bayern IDK Topologische Isolatoren.
[1] K. v. Klitzing, G. Dorda, and M. Pepper, Phys. Rev. Lett. 45, 494 (1980).

[2] T. Ando, A. B. Fowler, and F. Stern, Rev. Mod. Phys. 54, 437 (1982).

[3] K. von Klitzing, Rev. Mod. Phys. 58, 519 (1986).

[4] D. Többen, F. Schäffler, A. Zrenner, and G. Abstreiter, Phys. Rev. B 46, 4344 (1992).

[5] R. B. Laughlin, Phys. Rev. B 23, 5632 (1981).

[6] H. Aoki and T. Ando, Solid State Commun. 38, 1079 (1981).

[7] K. S. Novoselov, Z. Jiang, Y. Zhang, S. V. Morozov, H. L. Stormer, U. Zeitler, J. C. Maan, G. S. Boebinger, P. Kim, and A. K. Geim, Science 315, 1379 (2007).
[8] M. König, S. Wiedmann, C. Brüne, A. Roth, H. Buhmann, L. W. Molenkamp, X.-L. Qi, and S.-C. Zhang, Science 318, 766 (2007).

[9] B. Büttner, C. X. Liu, G. Tkachov, E. G. Novik, C. Brüne, H. Buhmann, E. M. Hankiewicz, P. Recher, B. Trauzettel, S. C. Zhang, and L. W. Molenkamp, Nat. Phys. 7, 418 (2011).

[10] D. A. Kozlov, Z. D. Kvon, N. N. Mikhailov, and S. A. Dvoretskii, JETP Lett. 100, 724 (2015).

[11] D. A. Kozlov, Z. D. Kvon, N. N. Mikhailov, S. A. Dvoretskii, S. Weishäupl, Y. Krupko, and J.-C. Portal, Appl. Phys. Lett. 105, 132102 (2014).

[12] B. A. Bernevig, T. L. Hughes, and S.-C. Zhang, Science 314, 1757 (2006). 
[13] E. G. Novik, A. Pfeuffer-Jeschke, T. Jungwirth, V. Latussek, C. R. Becker, G. Landwehr, H. Buhmann, and L. W. Molenkamp, Phys. Rev. B 72, 035321 (2005).

[14] C. Brüne, A. Roth, H. Buhmann, E. M. Hankiewicz, L. W. Molenkamp, J. Maciejko, X.-L. Qi, and S.-C. Zhang, Nat. Phys. 8, 486 (2012).

[15] J. I. Väyrynen, M. Goldstein, and L. I. L. Glazman, Phys. Rev. Lett. 110, 216402 (2013).

[16] M. König, M. Baenninger, A. G. F. Garcia, N. Harjee, B. L. Pruitt, C. Ames, P. Leubner, C. Brüne, H. Buhmann, L. W. Molenkamp, and D. Goldhaber-Gordon, Phys. Rev. X 3, 021003 (2013).

[17] M. Pakmehr, C. Bruene, H. Buhmann, L. W. Molenkamp, A. V. Stier, and B. D. McCombe, Phys. Rev. B 90, 235414 (2014).

[18] J. Ludwig, Y. B. Vasilyev, N. N. Mikhailov, J. M. Poumirol, Z. Jiang, O. Vafek, and D. Smirnov, Phys. Rev. B 89, 241406 (2014).

[19] Z. D. Kvon, E. B. Olshanetsky, E. G. Novik, D. A. Kozlov, N. N. Mikhailov, I. O. Parm, and S. A. Dvoretsky, Phys. Rev. B 83, 193304 (2011).

[20] B. Huckestein, Rev. Mod. Phys. 67, 357 (1995).

[21] E. Abrahams and P. Anderson, Phys. Rev. Lett. 42, 673 (1979).
[22] N. A. Dodoo-Amoo, K. Saeed, D. Mistry, S. P. Khanna, L. Li, E. H. Linfield, A. G. Davies, and J. E. Cunningham, J. Phys.: Condens. Matter 26, 475801 (2014).

[23] S. Koch, R. J. Haug, K. v. Klitzing, and K. Ploog, Phys. Rev. B 43, 6828 (1991).

[24] S. W. Hwang, H. P. Wei, L. W. Engel, D. C. Tsui, and A. M. M. Pruisken, Phys. Rev. B 48, 11416 (1993).

[25] H. P. Wei, D. C. Tsui, M. A. Paalanen, and A. M. M. Pruisken, Phys. Rev. Lett. 61, 1294 (1988).

[26] W. Li, C. L. Vicente, J. S. Xia, W. Pan, D. C. Tsui, L. N. Pfeiffer, and K. W. West, Phys. Rev. Lett. 102, 216801 (2009).

[27] J. J. Lin and J. P. Bird, J. Phys.: Condens. Matter 14, R501 (2002).

[28] T. Brandes, L. Schweitzer, and B. Kramer, Phys. Rev. Lett. 72, 3582 (1994).

[29] A. J. M. Giesbers, U. Zeitler, L. A. Ponomarenko, R. Yang, K. S. Novoselov, A. K. Geim, and J. C. Maan, Phys. Rev. B 80, 241411 (2009).

[30] Y. G. Arapov, S. V. Gudina, V. N. Neverov, S. M. Podgornykh, M. R. Popov, G. I. Harus, N. G. Shelushinina, M. V. Yakunin, N. N. Mikhailov, and S. A. Dvoretsky, Semiconductors 49, 1545 (2015). 